FRI0604

TRENDS OF INFORMED CONSENT FORMS FOR INDUSTRY-SPONSORED CLINICAL TRIALS IN RHEUMATOLOGY OVER A 17-YEAR PERIOD: READABILITY, AND ASSESSMENT OF PATIENTS' HEALTH LITERACYAND PERCEPTIONS

A.S. Meza-Lopez ${ }^{1}$, A. Gallardo-Castro ${ }^{1}$, A. Villarreal-Castellanos ${ }^{1}$, A. BarajasOchoa $^{2}$, J. Yanez ${ }^{3}$, L.P. Bustamante-Montes ${ }^{1}$, C. Ramos-Remus' ${ }^{1}$. ${ }^{1}$ Universidad Autonoma de Guadalajara; ${ }^{2}$ Unidad de Investigacion en Enfermedades CronicoDegenerativas, Guadalajara; ${ }^{3}$ Campus Ciudad de Mexico, Universidad Iberoamericana, Mexico, Mexico

Background: The therapeutic arsenal in rheumatology has improved drastically during the last 20 years with the availability of biological and synthetic diseasemodifying antirheumatic drugs. All these drugs required randomised controlled trials (RCTs) with thousands of rheumatic patients. All these patients went through the informed consent process and signed corresponding informed consent forms (ICFs).

Objectives: To assess trends in the length and readability of ICFs for industrysponsored RCTs over a 17 year period. Additionally, to assess the health literacy $(\mathrm{HL})$ and perceptions on ICFs among participants of current RCTs.

Methods: The grammatical readability (GR) of six pre-defined ICF sections (global, introduction, methods, risks/benefits, rights/responsibilities, and voluntary participation statement) of pivotal ICFs from industry-sponsored RCTs conducted at an outpatient rheumatology clinic during a 17 year period was assessed by using the INFLESZ instrument. Differences in GR were assessed by company, by disease and by study phase $(95 \% \mathrm{Cl}$ of the mean and proportions; statistical significance assumed if no overlap), and by the years ICFs were written (1999_-2005, 2006-2010, and 2011-2016; Kruskal-Wallis test). HL of patients was assessed with the SALHSA (inadequate, adequate) and S-TOFHLA (inadequate, marginal, adequate) instruments. Differences by age and schooling were determined by one-way ANOVA. Patient's perceptions and opinions on the ICF were assessed using a structured, self-reported, in-office questionnaire on an independent patient sample that had signed a pivotal ICF in the past 6 months.

Results: Thirty-nine ICFs about 22 drugs (18 biological or targeted synthetic DMARDs; 13 currently available on the market) from 13 pharmaceutical companies were analysed. The global mean readability was $57 \pm 3(95 \% \mathrm{Cl} 56-58)$, and all ICFs were categorised as either "somewhat difficult to read" or "average". Readability remained at these levels without significant changes from 1999 to 2016. The "somewhat difficult" reading score was significantly more frequent in the "rights and responsibilities" and in the "informed consent statement" sections $(p<0.001)$. The mean length of the ICFs written between 1999 and 2005 was $13 \pm 5$ pages, with a significant increase thereafter $(22 \pm 8$ pages, $p<0.01)$. Depending on the instrument, of 95 patients participating in the $\mathrm{HL}$ assessment, between $18 \%$ and $44 \%$ had limited $\mathrm{HL}$. Of 90 patients participating in the perceptions questionnaire, $84 \%$ reported understanding the ICF well. However, $2 \%-57 \%$ misunderstood basic concepts, including the study drug name, randomization and placebo. Conclusions: It seems that the considerable progress that has been made in medical research methods over time has not produced improvements in the process of informed consent in the industry-sponsored RCTs. The disparity between the readability of ICFs with patients' HL and their comprehension of ICFs continues, even after decades of attempts of regulatory agencies and numerous published suggestions.

Disclosure of Interest: None declared

DOI: 10.1136/annrheumdis-2018-eular.1565

\section{FRI0605 THE DEVELOPMENT PROCESS OF MOBILE HEALTH APPLICATIONS FOR SELF-MANAGEMENT IN PATIENTS WITH RHEUMATIC AND MUSCULOSKELETAL DISEASES IS HETEROGENEOUS AND OFTEN INCOMPLETE: RESULTS OF A SYSTEMATIC LITERATURE REVIEW}

A. Najm ${ }^{1}$, E. Nikiphorou ${ }^{2}$, L. Gossec ${ }^{3}$, F. Berenbaum ${ }^{4} .{ }^{1}$ Rheumatology department, Nantes University Hospital, Nantes, France; ${ }^{2}$ Academic Rheumatology Department, King's College London, London, UK, London, UK; ${ }^{3}$ Rheumatology Department, Sorbonne Universités, UPMC University Paris; ${ }^{4}$ Department of Rheumatology, Inflammation-Immunopathology-Biotherapy Department, Pierre and Marie Curie University, Saint-Antoine hospital, Sorbonne University, Paris, France

Background: Mobile health (mHealth) is exponentially growing in the current era, providing new potential and transforming the face of healthcare delivery. While the increasing availability of applications (apps) may enable people with rheumatic and musculoskeletal diseases (RMDs) to better self-manage their health, there is a general lack of evidence on ways to ensure appropriate development and evaluation of apps.
Objectives: To obtain an overview of existing mHealth apps for self-management in patients with RMDs, regarding content and development methods in particular, through a systematic literature review.

Methods: A search was performed up to December 2017 using EMBASE, Pubmed, Cochrane library, Web of Science and Psychinfo databases, with relevant key words and MeSH terms addressing three key domains: RMDs, self-management and mHealth. Double screening was performed on $15 \%$ of all abstracts against agreed inclusion criteria. For each publication relevant to an app for RMDs, the content, the aims, the target population of the apps and their development strategies were noted. The analysis was descriptive.

Results: Of 562 abstracts, 57 full text were screened. 39 articles were included in the analysis. 13 articles referred to an app linked to a connected device. Most of the apps targeted rheumatoid arthritis patients $(n=11)$, juvenile idiopathic arthritis $(n=7)$ and osteoarthritis $(n=8)$. The development process of the app was poorly described in $19 / 39(48.7 \%)$ of the studies (figure 1). Only $6 / 39(15.4 \%)$ included patients, and $14 / 39(35.9 \%)$ included health professionals or physicians in the development of such apps. Moreover, a qualitative phase occurred in only $6 / 39$ $(15.4 \%)$ of the cases. The app was tested and evaluated by physicians in $6 / 39$ $(15.4 \%)$. Patients were more frequently involved in app evaluation (29/39, $74.4 \%)$ but mostly indirectly through their adherence to the app. Only few of the apps published on were commercially available $(5 / 39,12.8 \%), 4 / 5$ are free. One app is currently under development.

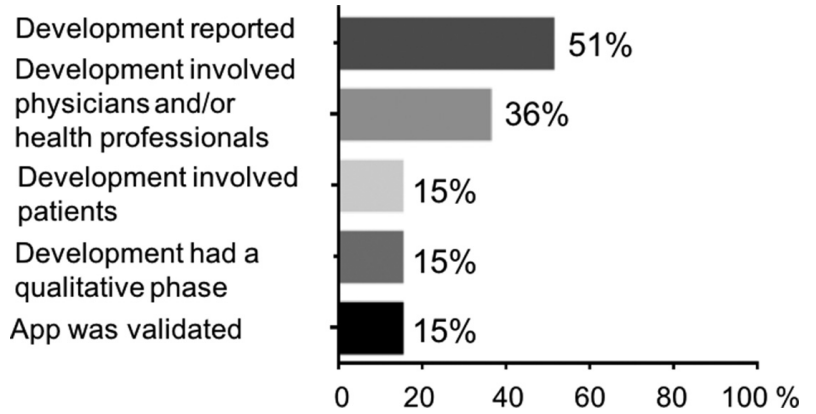

Abstract FRI0605 - Figure 1. Description of the development process of the apps in the 39 articles.

Conclusions: The development process of most apps was poorly described and potentially not satisfying in many studies. Despite patient willingness to use mHealth apps for self-management of their RMDs, a strong effort needs to be made to provide a standard and ensure quality and safety of newly-developed apps. This work will further inform EULAR points to consider for development, evaluation and implementation of mobile health applications for self-management of RMDs by patients.

Disclosure of Interest: None declared

DOI: 10.1136/annrheumdis-2018-eular.2129

\section{FRI0606 \\ THE LIFT STUDY'S DIRECT-TO-PATIENT DESIGN PROVIDES RELIABLE SELF-REPORTED DATA FROM LUPUS PATIENTS}

A. Cheeks, L. Borisov, K. Warren, R. Terbrueggen. DxTerity Diagnostics, Rancho Dominguez, USA

Background: Direct-to Patient (D2P) clinical studies that rely upon social media, mobile connectivity, wearables, self-collected patient samples, and patient reported outcome data have the potential to revolutionise health research by enabling population-scale studies without the cost and complexity of traditional multisite trials. D2P studies are particularly interesting for longitudinal studies where the burden of participants travelling to a central site is high; however, the quality and reliability of patient reported outcome data is unclear ${ }^{1}$.

Objectives: The study evaluated the accuracy of self-reported data from a subse of patients in a 1000+participant D2P lupus study (www.theliftstudy.com) by comparing patient-reported data to medical records as well as genomic testing of individuals using participant-collected fingerstick blood samples.

Methods: The study was reviewed and approved by a central IRB (WIRB Puyallup, WA). Subjects were recruited online across the U.S using social media. Electronically consented participants with self-reported lupus were asked to com plete online surveys about their disease and drug history as well as provide medical record review (MRR) consent. A third party firm was employed for MRR and an independent physician summarised the following information: 1) participant age 2) confirmation of lupus 3) current lupus medications and 4) current steroid medications. 